\title{
Economic benefits of the Mediterranean-style diet consumption in Canada and the United States
}

\author{
Mohammad M.H. Abdullah 1,2, Jason P.H. Jones ${ }^{3}$ and Peter J.H. Jones ${ }^{1,2}$ * \\ 'Department of Human Nutritional Sciences, University of Manitoba, Winnipeg, MB, Canada; ${ }^{2}$ Richardson Centre for \\ Functional Foods and Nutraceuticals (RCFFN), University of Manitoba, Winnipeg, MB, Canada; ${ }^{3}$ Department of \\ Agricultural Economics, Texas A\&M University, College Station, TX, USA
}

Abstract

Background: The Mediterranean-style diet (MedDiet) is an established healthy-eating behavior that has consistently been shown to favorably impact cardiovascular health, thus likely improving quality of life and reducing costs associated with cardiovascular disease (CVD). Data on the economic benefits of MedDiet intakes are, however, scarce.

Objective: The objective of this study was to estimate the annual healthcare and societal cost savings that would accrue to the Canadian and American public, independently, as a result of a reduction in the incidence of CVD following adherence to a MedDiet.

Design: A variation in cost-of-illness analysis entailing three stages of estimations was developed to 1) identify the proportion of individuals who are likely to adopt a MedDiet in North America, 2) assess the impact of the MedDiet intake on CVD incidence reduction, and 3) impute the potential savings in costs associated with healthcare and productivity following the estimated CVD reduction. To account for the uncertainty factor, a sensitivity analysis of four scenarios, including ideal, optimistic, pessimistic, and very-pessimistic assumptions, was implemented within each of these stages.

Results: Significant improvements in CVD-related costs were evident with varying MedDiet adoption and CVD reduction rates. Specifically, CAD \$41.9 million to 2.5 billion in Canada and US \$1.0-62.8 billion in the United States were estimated to accrue as total annual savings in economic costs, given the 'very-pessimistic' through 'ideal' scenarios.

Conclusions: Closer adherence to dietary behaviors that are consistent with the principles of the MedDiet is expected to contribute to a reduction in the monetary burdens of CVD in Canada, the United States, and possibly other parts of the world.

Keywords: cardiovascular disease; public health; healthy eating; nutrition economics; cost-of-illness analysis

Responsible Editor: Per Ole Iversen, Department of Nutrition, IMB, University of Oslo, Norway.

Received: II February 2015; Revised: 23 May 2015; Accepted: 26 May 20I5; Published: 24 June 2015

$\mathrm{T}$ he recent emergence of 'nutrition economics', the study of eating behaviors in the context of health economics, has offered better understanding of absolute and relative monetary impacts of health responses to dietary habits $(1,2)$. Dramatic rates in prevalence of cardiovascular diseases (CVDs), including coronary heart disease (CHD) and stroke, currently place great burdens on national economies worldwide. Globally, over 17 million, or $30 \%$, of all deaths were attributed to CVD in 2008 (3) and costing US $\$ 863$ billion in 2010 (4). Specific to North America, CVD accounted for $29 \%$ of all deaths in Canada in 2008 (5) and have been estimated to cost nearly CAD \$21 billion in annual healthcare costs based on 2005 monetary figures (6).
Also, about 600,000 Americans died of CHD alone in 2010 (7), with the direct and the indirect costs of the disease amounting to US $\$ 108.9$ billion (8).

Although the genetic predispositions cannot be ignored, it is well-acknowledged that lifestyle changes play the largest role within strategies to prevent, delay, or manage the progression of CVD, where healthy dietary patterns sit at the heart of the scenario (9). Outcomes of the presently largest 'hard' endpoint dietary intervention trial PREDIMED (10) recently confirmed a wealth of knowledge regarding the cardio-protective impacts of the largely plant-centered Mediterranean-style diet (MedDiet) (11). Specifically, 30 and $40 \%$ reductions in CHD and stroke, respectively, were evident with closer adherence to a 
MedDiet, in comparison to a low-fat control diet. In agreement, the Lyon Diet Heart Study, a secondary prevention trial, previously showed a $50 \%$ reduction in major cardiac events in CVD patients who followed a MedDiet (12). First proposed by Ancel Keys in the 1960s (13), the classical MedDiet is characterized by high intakes of fruit and vegetables, whole grains, legumes, and tree nuts; moderate intakes of cheese and yogurt, fish, poultry, and eggs; and low intakes of red and processed meat (14). This most widely accepted model of healthy nutritional patterns (15) also emphasizes extra-virgin olive oil (EVOO) as the principal source of daily fat intake and a glass of wine almost always during supper hours (14).

In spite of the considerable cardio-protective evidence from a myriad of epidemiological and intervention studies (16-22), the healthcare and societal benefits of the MedDiet consumption have yet to be fully monetized. The objective of this research was thus to estimate the potential annual savings in CVD-related economic costs in Canada and the United States, independently, under a MedDiet using multiple scenarios of intake.

\section{Methods}

In a conceptual framework design, a three-stage variation of cost-of-illness analysis was employed. This is an adapted version of a model that was originally presented by Malla et al. (23) and Gyles et al. (24) for economic benefits of canola oil and plant sterol consumptions, respectively. The first stage of the present model identified the proportion of individuals who are likely to adhere to a MedDiet in Canada and the United States based on the available nutrition literature. The second stage, again based on current medical literature, assessed the action of a MedDiet in terms of CVD incidence reduction. Finally, the third stage imputed the potential savings in healthcare- and society-related costs following the estimated CVD risk reduction. Figure 1 depicts the three stages of the economic model described in this article. In quest of the most robust predictions in a range of assumptions, four scenarios were generated within each stage of assessment using a sensitivity analysis as follows: ideal (best-case scenario), optimistic, pessimistic, and very pessimistic (worst-case scenario), as previously (24).

\section{Stage I: Assessment of the Mediterranean-style diet intake,} the 'adoption rate'

The adoption rate refers to the proportion of individuals who are likely to adhere to a MedDiet. In predicting the extent to which Canadians and Americans may be able to adopt this particular dietary pattern, presumably leading to improvements in cardiovascular health, and thus healthcare cost savings, an extrapolation of MedDiet score (MedScore) data of North American studies in the literature was utilized. Diverse in design, the MedScores are questionnaire-like scoring systems designed to assess

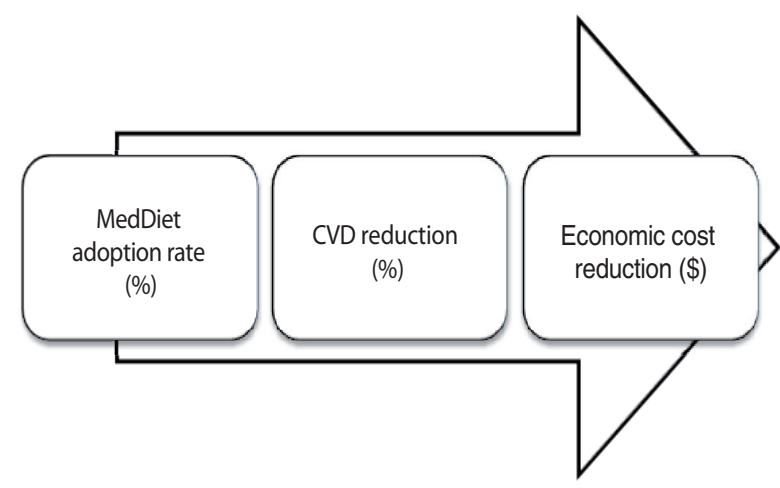

Fig. 1. The study's economic framework utilizing a variation of cost-of-illness analysis of three stages of estimations. Based on data from recent peer-reviewed literature and national databases, the first stage identified the proportions of individuals who are likely to adhere to a MedDiet in Canada and the United States, the second assessed the reported cardiovascular disease reduction rate following a MedDiet consumption, and the third stage imputed the potential reduction in economic costs associated with the estimated CVD incidence reduction. In covering a wide range of predictions, each stage constituted four scenarios of assumptions reflecting best- through worst-case scenarios as follows: ideal, optimistic, pessimistic, and very-pessimistic.

the habitual or recommended degrees of adherence to a MedDiet; some MedScores have been validated in different populations (25). Data from studies employing MedScores reveal adoption rates that range between 5 and $65 \%(26-35)$.

Stage 2: Evaluation of the reduction in incidence of CVDs following intakes of the Mediterranean-style diet

A myriad of cohort and randomized controlled studies with a wide spectrum of constructs have documented beneficial impacts of the MedDiet on CVD risk and/or mortality, with protection rates ranging between 10 and $70 \%(10,12,30,32,33,36-41)$. Akin to Stage 1 of this analysis, potential rates of reduction in incidence of CVD following intakes of the MedDiet were established based on data in the literature. Therefore, we focused on evidence deriving, as appropriate, from original and well-designed primary or secondary prevention trials that had involved male and female participants, investigated cardiovascular impacts of the MedDiet in its entirety (not merely single foods or nutrients of it), and reported adjusted relative risks (RR), hazard ratios (HR), or odds ratios (OR) for CVD with corresponding $95 \%$ confidence intervals. Data for this stage of the analysis were derived from studies conducted both in North America and Europe.

\section{Stage 3: Calculation of the potential savings in CVD-related economic costs}

In the third and final stage of this analysis, recent estimates of economic costs associated with CVD in Canada $(42,43)$ and the United States (44) were initially inflated to their 
2014 monetary equivalents, and then separately employed in the calculations of potential cost savings in the two countries under study. The point of view for this cost analysis is of particular importance due to the vastly different healthcare system funding schemes between Canada and the United States. The analysis provides estimates of direct cost savings from a provider prospective and an indirect saving component based on costs faced by the society. While the analysis provides critical components for a societal analysis, a complete evaluation from a societal viewpoint would require an economic evaluation of consumer choice and food market consequences. Drummond et al. (45) detail these various assessment perspectives and recommend appropriate methodologies for evaluating various healthcare programs. These methodologies are transferable to the current research for assessing the healthcare cost impact of reduced disease prevalence, where the authors provide generalized approaches for evaluating shared, direct, and indirect associated costs.

\section{Economic costs of CVDs in Canada}

Recent data on the economic costs attributed to CVD rates within Canada were extracted from the Economic Burden of Illness in Canada (EBIC) 2005-2008 report (42) and the National Health Expenditure Trends (NHEX) 1975-2013 report (43). Statistics Canada's Health Care Consumer Price Index (CPI) was subsequently used to translate the costs of CAD $\$ 12,188.8$ million in 2008 into the 2014 dollars of 13,042.1 million (Table 1).

Table 1. Summary of the economic costs attributed to CVD in Canada (CAD \$million)

\begin{tabular}{llrr}
\hline Year & & $2008^{\mathrm{a}}$ & \multicolumn{1}{c}{$2014^{\mathrm{b}}$} \\
\hline Direct costs & Hospitals & $5,068.0$ & $5,422.8$ \\
& Drugs & $4,272.7$ & $4,571.8$ \\
& Physicians & $2,352.0$ & $2,516.7$ \\
& Other directs $^{\mathrm{c}}$ & 134.1 & 143.5 \\
& Total direct & $1 \mathrm{I}, 826.8$ & $12,654.7$ \\
Indirect costs & Mortality & 92.4 & 98.9 \\
& Morbidity & 269.6 & 288.5 \\
& Total indirect & 362.0 & 387.3 \\
Total CVD costs & & $12,188.8$ & $13,042.1$ \\
\hline
\end{tabular}

${ }^{\mathrm{a}}$ From the Economic Burden of Illness in Canada (EBIC) 2005-2008 report (42). ${ }^{b}$ Current dollars based on adjustments according to Statistics Canada's Health Care Consumer Price Index. 'Comprises costs for 'Other Professionals' (chiropractors, physiotherapists, private duty nurses, etc.) and 'Other Health Spending' (home care, medical transportation, etc.) by the National Health Expenditure Trends (NHEX) 1975-2013 report (43), where the total 'Other Direct' costs for all diseases in 2008 equaled CAD $\$ 53,022$.I million. Percentage of CVD relative to total 'Other Direct' costs within the EBIC 1998 report (46) of $0.3 \%$ was used to estimate our 2008's 'Other directs' monetary figure of CAD $\$ 134.1$ million.
As presented by EBIC 2005-2008 and summarized in Table 1, the CVD-related economic costs in Canada were divided into direct and indirect categories. The former represents costs associated with hospital operations, medications, and physicians' and other healthcare professionals' payments, whereas the latter deals with cost estimates that relate to reduced productivity due to mortality, illness, and disabilities. Seeking closer estimations on cost savings that would accompany each $1 \%$ reduction in CVD incidence, components of the direct and indirect categories were assessed individually, similar to previously (24).

A 1999 cost analysis study (47) suggested that $84 \%$ of hospitalization costs are 'fixed' (i.e. salaried labor, buildings, and equipment), thus not affected by the number of disease cases, whereas $16 \%$ are 'variable' (i.e. medications and supplies). Our economic model thus presumed that each $1 \%$ reduction in the incidence of CVD would logically be followed by a $0.16 \%$ reduction in hospital costs. Notably, this projection is conservative when compared to the $25-42 \%$ variable hospitalization cost figure previously reported by other authors (48, 49). Given that fewer CVD cases would, as well, sensibly lead to fewer drugs prescribed for treatment, it was presumed that each $1 \%$ reduction in CVD incidence would be accompanied by a comparative $1 \%$ reduction in cost of medications. The same concept was applied to the physician care component of the direct cost category, where fewer visits to physician offices would in theory result in lower billing-related costs; therefore, a $1 \%$ lower CVD rate was assumed to lower this category also by $1 \%$. The last component of the direct cost category, the 'other directs', comprises costs for other institutions, other professionals (dental services, vision care services, and other), capital, public health, and other health spending (e.g. health research) (42). Some of these medical services were logically assumed not to constitute a significant part of the CVD-related cost burden and were hence not included in our valuation. The other direct health expenditure totals were included in the EBIC 20052008 report; however, they were not attributed by EBIC categories, such as certain sex, age group, or a particular diagnosis. Our model thus relied on data from the NHEX 1975-2013 (43) report to assume that a 1\% decrease in the incidence of CVD would translate into a $0.19 \%$ decrease in costs of the 'other directs' component. This value was based on estimations of services by the NHEX 1975-2013 'Other' (5.4\%), within the 'Other Professionals' category, defined as costs for chiropractors, physiotherapists, private duty nurses, and others, as well as services by the 'Other' $(13.8 \%)$ within the 'Other Health Spending' category, defined as costs for home care, medical transportation, training of health workers, and others. Finally, costs that are associated with the two components of the indirect category were, on the other hand, predicted to decline in proportion to the CVD incidence reduction, 
based on the assumption that as the number of individuals with CVD decreases, a reduction in the premature mortality and disability rates would follow proportionately (Table 2).

\section{Economic costs of CVDs in the United States}

Calculations of the potential savings in economic costs associated with CVD for the United States were conducted in a manner similar to that of Canada, but using separate data sets. Go et al. (44) published a report on behalf of the American Heart Association (AHA), incorporating the Medical Expenditure Panel Survey data with current demographic figures to produce the direct (hospital, home healthcare, and prescribed medicines) and indirect (lost productivity/mortality) costs associated with CVD rates in the United States. There, increased detail on hospital costs (i.e. inpatient, emergency, and outpatient) was available, with physician costs included in the 'Hospital outpatient or office-based provider visits' category. We used monetary adjustments according to the US Department of Labor's Health Care CPI to translate the CVD-associated costs of US $\$ 315.4$ billion in 2010 into $\$ 346.9$ billion in 2014 (Table 3). Similar to the Canadian context, the corresponding percentage decreases from a $1 \%$ reduction in CVD were estimated individually. All hospital-related costs were assumed to only decrease by their respective approximated $0.16 \%$ 'variable' costs, as discussed earlier. Home healthcare is in theory dependent on disease prevalence; thus, a 1:1 relationship between CVD prevalence and costs was assumed, which was also the case with

Table 2. Summary of the cost reductions corresponding to $1.0 \%$ reduction in incidence of CVD within Canada and the United States

\begin{tabular}{|c|c|c|c|c|}
\hline \multirow[b]{3}{*}{ Direct reduction } & \multicolumn{4}{|c|}{ Cost reduction (\%) } \\
\hline & \multicolumn{2}{|l|}{ Canada } & \multicolumn{2}{|l|}{ United States } \\
\hline & Hospitals & 0.16 & Hospital, inpatient ${ }^{\mathrm{b}}$ & 0.16 \\
\hline & Drugs & 1.00 & Hospital, emergency ${ }^{c}$ & 0.16 \\
\hline & Physicians & 1.00 & Hospital, outpatient $^{\text {d }}$ & 0.16 \\
\hline & Other directs ${ }^{\mathrm{a}}$ & 0.19 & Home healthcare & 1.00 \\
\hline & & & Prescribed medicines & 1.00 \\
\hline \multirow[t]{2}{*}{ Indirect reduction } & Mortality & 1.00 & Lost productivity/ & 1.00 \\
\hline & Morbidity & 1.00 & mortality & \\
\hline
\end{tabular}

All hospital figures are based on the estimation that $16 \%$ of hospitalization costs are variable (i.e. medications and supplies) and $84 \%$ are fixed

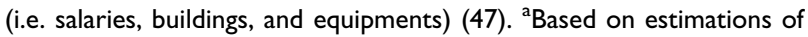
services by the National Health Expenditure Trends (NHEX) 1975-2013 'Other' (5.4\%), within the 'Other Professionals' category and 'Other' (13.8\%) within the 'Other Health Spending' category (43). ' ${ }^{\circ}$ Refers to room and board, and all diagnostic and laboratory costs. 'Refers to diagnostic and laboratory costs. Includes office-based provider visits, laboratory costs, and visits to medical providers. The US categories are from Go et al. (44).
Table 3. Summary of the economic costs attributed to CVD in the United States (US \$billion)

\begin{tabular}{llrr}
\hline Year & & $2010^{\mathrm{a}}$ & ${ }^{2014^{\mathrm{b}}}$ \\
\hline Direct costs & Hospital, inpatient $^{\mathrm{c}}$ & 98.0 & 107.3 \\
& Hospital, emergency $^{\mathrm{d}}$ & 8.7 & 9.6 \\
& Hospital, outpatient $^{\mathrm{e}}$ & 42.2 & 46.4 \\
& Home healthcare & 11.7 & 12.9 \\
& Prescribed medicines & 32.8 & 36.1 \\
& Total direct & 193.4 & 212.7 \\
Indirect costs & Lost productivity/mortality & 122.0 & 134.2 \\
Total CVD costs & & 315.4 & 346.9 \\
\hline
\end{tabular}

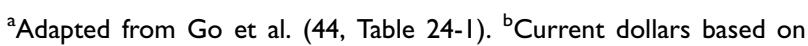
monetary adjustments according to the healthcare category of the US Department of Labor's Health Care Consumer Price Index. 'Refers to room and board, and all diagnostic and laboratory costs. ${ }^{\mathrm{d}}$ Refers to diagnostic and laboratory costs. Includes office-based provider visits, laboratory costs, and visits to medical providers.

medications as well as the only indirect cost component, 'lost productivity/mortality' (Table 2).

\section{Results}

\section{Mediterranean-style diet adoption rate}

Of the epidemiological and dietary intervention studies that have revealed varying levels of adherence to the MedDiet in Mediterranean and non-Mediterranean regions (26-35), MedScore findings ( $\%$ of lowest and highest self-reported intakes) specific to three North American studies were of particular interest $(26,31,32)$. A 12-week nutrition intervention advocating intakes of MedDiet in a 'real life' situation observed adoption rates of 48 and $65.5 \%$ in 73 French-Canadian women at baseline and endpoint, respectively (26). This study employed a global MedScore based on the Mediterranean diet pyramid (Oldways Preservation and Exchange Trust) of 11 components, including grains, fruits, vegetables, legumes, nuts and seeds, olive oil, dairy products, fish, poultry, eggs, and sweets and red meat/processed meat to evaluate the adherence to the recommended dietary pattern based on group and individual sessions. In the NHANES III study, involving 13,197 individuals in the United States, highest MedDiet adoption rates were reported by 27.9 and $39.5 \%$ of $<45$ - and $\geq 45$-year-old males, and 32.2 and $40.0 \%$ of pre- and postmenopausal females, respectively (31). In the same study, 37.5 and $30.0 \%$ of $<45$ - and $\geq 45$-year-old males, and 35.7 and $30.7 \%$ of pre- and postmenopausal females, respectively, reported lowest adoption rates. This study employed the scoring methodology by Panagiotakos et al. (50) of 11 components, including non-refined cereals and products, potatoes, fruits, vegetables, legumes, fish, red meat and products, poultry, full-fat dairy products, olive oil, and alcoholic beverages. 
Finally, in the NOMAS cohort (32), 25 and $14 \%$ of the 2,568 participants, respectively, scored highest and lowest on the employed MedDiet scoring methodology by Trichopoulou et al. (27) of nine components, including dairy, meat, fruit, vegetables (excluding potatoes), legumes, cereals (all cereals including refined and whole grain), fish, alcohol, and monounsaturated to saturated fatty acid (MUFA/SFA) ratio (32). Other North American studies reported similar adoption rates of $14-56 \%(51-53)$. In establishing MedDiet adoption rates in our sensitivity analysis that would reflect realistic ranges of intakes in a North American state, conservative rates of 50, 25, 15, and $5 \%$ were chosen to, respectively, reflect the potential ideal, optimistic, pessimistic, and very-pessimistic scenarios (Table 4).

\section{CVD reduction}

In determining the ideal scenario of CVD incidence reduction with intakes of the MedDiet based on previous research $(10,12,30,32,33,36-41)$, our economic model considered the $60 \%$ reduction observed in participants with the highest MedDiet adherence in the Spanish SUN cohort, which followed 13,609 individuals over 4.9 years (33). An optimistic 30\% primary endpoint event reduction was chosen based on data of the group assigned to a MedDiet with EVOO in the PREDIMED trial (10). This was consistent with the $33 \%$ decreased risk rate observed in a cluster of four markers for CVD (hypertension, diabetes, obesity, and hypercholesterolemia) among individuals who reported higher MedDiet intakes in the cross-sectional data of PREDIMED, involving 3,204 Spanish participants (30). A pessimistic incidence reduction assumption of $20 \%$ was considered based on the 12-year follow-up data from the Dutch contribution to the EPIC cohort, which involved 40,011 participants (41). This was similar to the $15 \%$ lower risk previously reported with the MedDiet group of the French MediRIVAGE study, a 3-month parallel design dietary intervention involving 212 participants (38). Data from two cohorts in the United States $(32,40)$ showed similar protection rates of $20-22 \%$ and fell well within our model assumptions for the optimistic and pessimistic scenarios. Finally, a very-pessimistic CVD risk reduction scenario of $10 \%$ was chosen based on the findings from the

Table 4. Summary of the sensitivity analysis (\%)

\begin{tabular}{lcccc}
\hline & \multicolumn{4}{c}{ Scenario } \\
\cline { 2 - 5 } & Ideal & Optimistic & Pessimistic & Very pessimistic \\
\hline Adoption rate & 50.0 & 25.0 & 15.0 & 5.0 \\
$\begin{array}{l}\text { CVD incidence } \\
\text { reduction }\end{array}$ & 60.0 & 30.0 & 20.0 & 10.0 \\
\hline
\end{tabular}

CVD, cardiovascular disease.
Table 5. Potential cost savings attributed to the reduction in CVD with varying adoption rates of MedDiet in Canada (CAD \$million)

\begin{tabular}{lrrrr}
\hline & \multicolumn{4}{c}{ Scenario $^{\mathrm{a}}$} \\
\cline { 2 - 5 } & Ideal & Optimistic & Pessimistic & Very pessimistic \\
\hline Direct savings & & & & \\
$\quad$ Hospitals & 260.3 & 65.1 & 26.0 & 4.3 \\
Medications & $1,371.5$ & 342.9 & 137.2 & 22.9 \\
$\quad$ Physicians & 755.0 & 188.7 & 75.5 & 12.6 \\
$\quad$ Other directs & 8.3 & 2.1 & 0.8 & 0.1 \\
Total direct & $2,395.1$ & 598.8 & 239.5 & 39.9 \\
Indirect savings & & & & \\
$\quad$ Mortality & 29.7 & 7.4 & 3.0 & 0.5 \\
$\quad$ Morbidity & 86.5 & 21.6 & 8.7 & 1.4 \\
Total indirect & 116.2 & 29.1 & 11.6 & 1.9 \\
Total savings & $2,511.3$ & 627.8 & 251.1 & 41.9 \\
\hline
\end{tabular}

${ }^{\text {a }}$ The ideal scenario represents a best-case estimate of potential economic cost savings when $50 \%$ of the population follows a MedDiet and shows $60 \%$ reduction in cardiovascular disease (CVD). The optimistic scenario is a medium- to short-term pragmatic estimate of potential cost savings when $25 \%$ of the population adopts a MedDiet and experiences $30 \%$ reduction in CVD. The pessimistic scenario is a practical short- to medium-term estimate of cost savings that could follow a MedDiet adherence among $15 \%$ of the population with $20 \%$ reduction in CVD. The very-pessimistic scenario represents a worst-case estimate with only $5 \%$ of the population making the dietary change and showing $10 \%$ reduction in CVD.

CARDIO2000 multicenter, case-control study that involved 418 CVD patients and 303 hypertensive controls in Greece (36). The majority of these studies estimated the MedDiet consumption based on the scoring methodology by Trichopoulou et al. (27) as described above. A summary of the CVD reduction rates for Canada and the United States is shown in Table 4.

\section{CVD-related economic cost reduction}

Our sensitivity analysis predicted total annual economic cost savings in Canada that ranged between CAD \$2,511.3 and $\$ 41.9$ million for the ideal through very-pessimistic scenarios (Table 5). Specifically, while the direct cost savings ranged between CAD \$2,395.1 and \$39.9 million, the indirect cost savings accounted for CAD \$116.2 to $\$ 1.9$ million annually. These estimates reflect the most recent comprehensive burden of illness data available in Canada, in conjunction with a thorough analysis of the peer-reviewed literature concerning the MedDiet and CVD incidence relationship. The wide range of economic cost savings can be primarily attributed to the variability in the latter parameter, given the consideration of such a large number of studies to determine this relationship.

The potential annual economic cost savings for the United States are presented in Table 6. Across the range 
Table 6. Potential cost savings attributed to the reduction in CVD with varying adoption rates of MedDiet in the United States (US \$ billion)

\begin{tabular}{lrccc}
\hline & \multicolumn{3}{c}{ Scenario $^{\mathrm{a}}$} \\
\cline { 2 - 5 } & & & & Very \\
& Ideal & Optimistic & Pessimistic & pessimistic \\
\hline Direct savings & & & & \\
Hospital, inpatient & 5.2 & 1.3 & 0.5 & 0.1 \\
Hospital, emergency & 0.5 & 0.1 & 0.0 & 0.0 \\
Hospital, outpatient & 2.2 & 0.6 & 0.2 & 0.0 \\
Home healthcare & 3.9 & 1.0 & 0.4 & 0.1 \\
Prescribed medicines & 10.8 & 2.7 & 1.1 & 0.2 \\
Total direct & 22.5 & 5.6 & 2.3 & 0.4 \\
Indirect savings & & & & \\
$\quad$ Lost productivity/ & 40.3 & 10.1 & 4.0 & 0.7 \\
$\quad$ mortality & & & & \\
Total savings & 62.8 & 15.7 & 6.3 & 1.0 \\
\hline
\end{tabular}

${ }^{a}$ The ideal scenario represents a best-case estimate of potential economic cost savings when $50 \%$ of the population follows a MedDiet and shows $60 \%$ reduction in cardiovascular disease (CVD). The optimistic scenario is a medium- to short-term pragmatic estimate of potential cost savings when $25 \%$ of the population adopts a MedDiet and experiences $30 \%$ reduction in CVD. The pessimistic scenario is a practical short- to medium-term estimate of cost savings that could follow a MedDiet adherence among $15 \%$ of the population with $20 \%$ reduction in CVD. The very-pessimistic scenario represents a worst-case estimate with only $5 \%$ of the population making the dietary change and showing $10 \%$ reduction in CVD.

of MedDiet adoption and CVD reduction scenarios, from ideal to very-pessimistic, the total cost savings were estimated as US $\$ 62.8-1.0$ billion for 2014 inflation-adjusted dollars, with direct and indirect estimates of US \$22.5-0.4 and \$40.3-0.7 billion, respectively. In all scenarios, the dollar savings associated with the reduction in CVD-related deaths and the subsequent productivity recoupment (indirect estimates) remained a greater benefit relative to the associated healthcare cost savings (direct estimates). Reduction in prescribed medicine expenditures in particular accounted for nearly half of the direct savings in the total healthcare costs.

\section{Discussion}

Employing data from the established scientific literature and up-to-date monetary figures, this economic analysis assessed the potential annual dollar savings in CVDrelated costs within the North American healthcare systems following adherence to a MedDiet. Evidence of considerable opportunities for economic cost savings is provided. Specifically, if between 5 and $50 \%$ of the Canadian or American populace follow a MedDiet, an estimated CAD $\$ 41.9$ million to $\$ 2.5$ billion in Canada, or US $\$ 1.0$ to $\$ 62.8$ billion in the United States, would accrue as total annual savings in direct and indirect costs, given the 'very-pessimistic' through 'ideal' scenarios. While the former 'worst-case' scenario reflects a situation where the general public's MedDiet adoption rate is lowest and poorly translated to beneficial impacts on CVD risk, the latter 'best-case' scenario is attainable with higher adoption rates and larger reductions in CVD over the long run. Cost savings associated with the 'pessimistic' and 'optimistic' scenarios, ranging between CAD \$251.1 and \$627.8 million in Canada, and US $\$ 6.3$ and $\$ 15.7$ billion in the United States, represent short- to medium-term benefits of the MedDiet and are thus realistically viewed as most probable.

The economic cost of disease is generally broken down into direct and indirect components, respectively covering services by the healthcare system and the loss of productivity to society arising from morbidity and premature mortality. The present economic model considered both these components, with some differences between the two countries under study. A particularly important issue regarding international comparability of the study's metrics relates to the differing estimation techniques for productivity effects. These are known to be estimated by one of three approaches. Whereas both the human capital approach and the friction cost approach place monetary value on the productivity costs, the approach recommended by the US Panel on Cost-Effectiveness in Health and Medicine includes part of the value of these costs in the assessment of health outcomes (54). As our data show, the human capital approach implemented by the AHA for the US context, using a 3\% discount rate on future earnings, yielded larger estimates relative to the equivalent indirect costs found in Canada, as well as the magnitude relative to direct costs. The indirect costs in the Canadian EBIC 2005-2008 report implemented the friction cost approach, accounting for surplus employment and the ability to replace a worker. The friction cost approach assumes that after a certain amount of time, referred to as the friction period, worker replacement occurs in addition to mechanisms that compensate for the workers absence. Conversely, the human capital approach values mortality and morbidity through decreased productivity during a workers employed lifetime, often valued by a wage rate. Most noted by critics to the human capital approach are the unrealistic assumption of 'full employment' and the exclusion of leisure time valuation (55). Pritchard and Sculpher (54) critically review and compare both these approaches, concluding relative merit of each.

In spite of the extensively documented cardio-protective properties of the MedDiet, merely a handful of costeffectiveness reports have forecasted its potential economic benefits (15). Of those reports, to our knowledge, only one evaluated the healthcare costs associated with a MedDiet, relative to a non-MedDiet intakes, based on 
estimations of a 10-year risk of developing CHD in 3,042 healthy Greek men and women (56). There, per patient hospitalization costs of $€ 690$ were estimated to be the result of a CHD event. Participants who were 'closer' to the MedDiet showed a $43 \%(\mathrm{OR}=0.57,95 \%$ CI $0.38-0.86)$ lower likelihood of having a 10 -year risk of over $10 \%$, with a total healthcare cost of $€ 35.880$, relative to $€ 336.720$ in those who were 'away' from the MedDiet. Our observation confirms such an association in the context of a North American setting and calls for an analysis of the outcomes from both the basic research and behavioral nutrition viewpoints.

By design, the conservative calculations presented in this study focused on the cost savings specifically associated with reductions in CVD incidence upon adherence to a MedDiet; however, in reality, other economic benefits are likely to follow higher adoption rates of such a healthyeating pattern. Such benefits would, for instance, accrue to other agribusiness stakeholders who would respond to a greater demand by the informed general public with a variety of healthy-style plant- and animal-based lines of products that may constitute parts of the typical MedDiet. Furthermore, given that it is an 'eating habit' that constellates a number of healthy foods and nutrients, the MedDiet has been shown to lead to improvements in other public health and wellness priorities, such as cancer (57), and is certainly expected to direct similar dollar savings on costs associated with such a major health concern.

The first step towards a MedDiet-associated health and economic benefits is the attainment of a higher adoption rate. This factor is largely influenced by the consumer's food decisions in the marketplace, which are in turn driven by the utility maximization. In economic theory, utility is defined as an unobservable behavioral metric of the satisfaction that the consumers sense with their choice of goods or services. Many factors affect how consumers maximize their utility, or in terms of dietary settings, choose what they eat, including habit, culture, convenience, taste, cost, personal values, and health (58). Research in food marketing also draws attention to a number of the so-called 'external cues' that may affect eating habits (59). When consumers are made aware of these factors and sustain behaviors of consuming healthy dietary patterns, health benefits along with concordant economic savings are expected to arise. Specific to the MedDiet, a free-living study promoting higher adherence among a cohort of Canadian participants demonstrated significant reductions in circulating total cholesterol levels and body mass index, two established biomarkers of cardiovascular health, with increased MedDiet adoption rates (26). The scenario analysis of our model sets the groundwork for similar studies in the future, allowing for the computation of economic savings for those wishing to explore the possible avenues and implications of promoting the MedDiet. Dynamics have important implications on the results of this study when considering the adoption of a novel diet. As it cannot be assumed that a MedDiet adoption will be instantaneous, the scenario approach reflects uncertainty in the adoptions of timing and extent. Determining savings across a continuum of adoption paths and CVD reduction rates will provide an opportunity of comparing the costs and benefits of such promotion programs.

The value of dollar savings observed with the MedDiet adoption and CVD risk reduction scenarios in this sensitivity analysis, specifically with the ideal scenario, but also other scenarios, underscores the synergistic responsibilities of stakeholders such as food industrialists and marketers, policy makers, educators, researchers, and healthcare professionals in establishing healthy food environments and attitudes. For instance, creating and advertising lines of healthy food products, appealing to the consumers, by the food industry and marketing sectors should be accompanied by clear guidelines by policy makers and sufficient messages of knowledge by educators and the media to the general public regarding the intrinsic health-related economic importance of those food products. Such action would essentially rely on a body of evidence from well-designed dietary studies and, most important, economic models such as the one of the present study.

This economic model possesses a number of strengths. It is to our knowledge the first to examine the potential economic savings attributed to lower incidence of CVD with adherence to MedDiet in North America. The model template was derived in part from those utilized in Malla et al. (23) and Gyles et al. (24), hence share some similarities. However, the nature of the dietary intervention proposed is distinctly different particularly in that it represents a combination of dietary ingredients in the current analysis compared to single entities in previous research. Investigating whole foods and dietary behaviors in their entirety rather than individual nutrients is logically, and evidently, better in capturing a more realistic view into the potential health and economic benefits for societies. As such, the downstream implications of the dietary transformation are highly disparate for the present MedDiet intervention versus canola and plant sterol interventions. Another strength of the present analysis relates to the joint discussion of Canada and the United States. While it is true that the current dramatic rates of CVD place similar manner of burdens on national resources of the affected societies worldwide, the disease and cost-saving estimates from non-Mediterranean regions hold a special importance. The vast majority of studies associating MedDiet consumption with cardiovascular health benefits emerge from countries of the Mediterranean basin where independent lifestyle factors are likely an influential contributor. This is not the case in 
countries with the so-called typical North American diet, or Western diet, where other lifestyle factors are unlikely to contribute to a MedDiet intervention's predicted health benefits. The present analysis hence enabled the assessment of benefits of a MedDiet adoption in the absence of contributing 'healthy' lifestyle factors that typically accompany this eating pattern in Mediterranean countries. This joint discussion also allowed for establishing a MedDiet's economic benefit in two countries that differ in population size and constructs of healthcare systems; a universal healthcare coverage in Canada and a combination of public and private healthcare efforts in the United States. Additionally, by design, the sensitivity analysis relied on relevant evidence from the established literature and enabled coverage of a range of assumptions for most robust predictions. And, the final economic figures were made possible with employment of the most recent healthcare costs available while adjusting for current monetary values. This model is also subject to some limitations, however. There is no one specific definition for the 'Mediterranean diet', given the broad food system and cultural differences in countries where it is typically adopted; 'Mediterranean-style diet', as used and defined in this article, is hence a more descriptive term of such an eating pattern. Also, other lifestyle factors in the Mediterranean regions might, as stated previously, have contributed to shaping the reported health benefits of the MedDiet beyond the dietary pattern per se. Estimations of the potential adoption and CVD reduction rates in the current analysis were thus based on data from studies in North America and various countries, respectively. In addition, from both the economic and nutrition standpoints, food substitution remains an important issue that was not captured in the analysis, impacting relative quality of health and product prices. It is also important to note that this approach is conservative regarding the responsiveness of hospital-related resource allocation, whereas in the long-term fixed costs are able to adjust. Future research ought to take these points into consideration and include such a dynamic economic construct within the design of carefully controlled human intervention trials of both short and long durations.

\section{Conclusion}

The present novel monetary modeling exercise sheds light on the economic importance of the MedDiet, beyond its well-established health impacts. This may aid in improving policies to increase the consumers' and policy makers' general knowledge of the concept of healthy eating, leading to better decisions in the marketplace, and thus improved quality of life. Such dietary guidance directives are expected to ultimately contribute to optimizing the sustainability of healthcare provisions and the handling of national resources as a result of lower social and monetary burdens associated with CVD in Canada, the United States, and possibly other parts of the world.

\section{Authors' contribution}

MMHA formulated the research question, designed the economic model, and wrote the manuscript. JPHJ assisted in providing sources of latest economic figures, in interpreting monetary data, and in shaping parts of the manuscript. PJHJ had primary responsibility for the final content. All authors reviewed the manuscript critically and approved its final version.

\section{Acknowledgements}

We acknowledge Ms. Jacqueline Dugas, Health Economist at the Public Health Agency of Canada (PHAC), for providing us with the latest and at the time yet to be released Economic Burden of Illness in Canada (EBIC) 2005-2008 data on healthcare costs associated with cardiovascular disease. We also thank Ms. Jian Gao, Senior Analyst (Health Expenditures) at the Canadian Institute for Health Information (CIHI), for providing us with clarifications with regard to specific economic figures in the National Health Expenditure Trends (NHEX) 1975-2013 report.

\section{Conflict of interest and funding}

The authors declare no conflict of interest in association with the content of the present article.

\section{References}

1. Lenoir-Wijnkoop I, Dapoigny M, Dubois D, van Ganse E, Gutierrez-Ibarluzea I, Hutton J, et al. Nutrition economics characterising the economic and health impact of nutrition. Br J Nutr 2011; 105: 157-66.

2. Gyles CL, Lenoir-Wijnkoop I, Carlberg JG, Senanayake V, Gutierrez-Ibarluzea I, Poley MJ, et al. Health economics and nutrition: a review of published evidence. Nutr Rev 2012; 70: 693-708

3. World Health Organization (2011). Global status report on noncommunicable diseases 2010. Geneva, Switzerland: WHO.

4. Bloom DE, Cafiero ET, Jané-Llopis E, Abrahams-Gessel S, Bloom LR, Fathima S, et al. The Global Economic Burden of Noncommunicable Diseases. Geneva, Switzerland: World Economic Forum; 2011.

5. Statistics Canada (2011). Mortality, summary list of causes 2008. Ottawa, ON: Statistics Canada.

6. The Conference Board of Canada (2010). The Canadian heart health strategy: risk factors and future cost implications report. Ottawa, ON: The Conference Board of Canada.

7. Murphy SL, Xu J, Kochanek KD. Deaths: final data for 2010 . Natl Vital Stat Rep 2013; 61: 1-117.

8. Heidenreich PA, Trogdon JG, Khavjou OA, Butler J, Dracup K, Ezekowitz MD, et al. Forecasting the future of cardiovascular disease in the United States: a policy statement from the American Heart Association. Circulation 2011; 123: 933-44.

9. Hu FB, Stampfer MJ, Manson JE, Grodstein F, Colditz GA, Speizer FE, et al. Trends in the incidence of coronary heart disease and changes in diet and lifestyle in women. $\mathrm{N}$ Engl $\mathbf{J}$ Med 2000; 343: 530-7. 
10. Estruch R, Ros E, Salas-Salvado J, Covas MI, Corella D, Aros F, et al. Primary prevention of cardiovascular disease with a Mediterranean diet. N Engl J Med 2013; 368: 1279-90.

11. Sofi F, Abbate R, Gensini GF, Casini A. Accruing evidence on benefits of adherence to the Mediterranean diet on health: an updated systematic review and meta-analysis. Am J Clin Nutr 2010; 92: 1189-96.

12. de Lorgeril M, Salen P, Martin JL, Monjaud I, Delaye J, Mamelle N. Mediterranean diet, traditional risk factors, and the rate of cardiovascular complications after myocardial infarction: final report of the Lyon Diet Heart Study. Circulation 1999; 99: 779-85.

13. Keys A, Menotti A, Karvonen MJ, Aravanis C, Blackburn H, Buzina $\mathrm{R}$, et al. The diet and 15 -year death rate in the seven countries study. Am J Epidemiol 1986; 124: 903-15.

14. McKeown PP, Logan K, McKinley MC, Young IS, Woodside JV. Session 4: CVD, diabetes and cancer: evidence for the use of the Mediterranean diet in patients with CHD. Proc Nutr Soc 2010; 69: 45-60.

15. Saulle R, Semyonov L, La Torre G. Cost and cost-effectiveness of the Mediterranean diet: results of a systematic review. Nutrients 2013; 5: 4566-86.

16. Bogani P, Galli C, Villa M, Visioli F. Postprandial antiinflammatory and antioxidant effects of extra virgin olive oil. Atherosclerosis 2007; 190: 181-6.

17. Sofi F, Cesari F, Abbate R, Gensini GF, Casini A. Adherence to Mediterranean diet and health status: meta-analysis. BMJ 2008; 337: a1344.

18. Esposito K, Maiorino MI, Ceriello A, Giugliano D. Prevention and control of type 2 diabetes by Mediterranean diet: a systematic review. Diabetes Res Clin Pract 2010; 89: 97-102.

19. Kastorini CM, Milionis HJ, Esposito K, Giugliano D, Goudevenos JA, Panagiotakos DB. The effect of Mediterranean diet on metabolic syndrome and its components: a metaanalysis of 50 studies and 534,906 individuals. J Am Coll Cardiol 2011; 57: 1299-313.

20. Sofi F, Macchi C, Abbate R, Gensini GF, Casini A. Mediterranean diet and health. Biofactors 2013; 39: 335-42.

21. Esposito K, Kastorini CM, Panagiotakos DB, Giugliano D. Mediterranean diet and metabolic syndrome: an updated systematic review. Rev Endocr Metab Disord 2013; 14: 255-63.

22. Sofi F, Macchi C, Abbate R, Gensini GF, Casini A. Mediterranean diet and health status: an updated meta-analysis and a proposal for a literature-based adherence score. Public Health Nutr 2014; 17: 2769-82.

23. Malla S, Hobbs J, Perger O. Estimating the value of health care savings from trans fat-free canola in Canada. Food Econ - Acta Ag Scandi. Sec C 2005; 2: 175-84.

24. Gyles CL, Carlberg JG, Gustafson J, Davlut DA, Jones PJ. Economic valuation of the potential health benefits from foods enriched with plant sterols in Canada. Food Nutr Res 2010; 54. doi: 10.3402/fnr.v54i0.5113.

25. Bach A, Serra-Majem L, Carrasco JL, Roman B, Ngo J, Bertomeu I, et al. The use of indexes evaluating the adherence to the Mediterranean diet in epidemiological studies: a review. Public Health Nutr 2006; 9: 132-46.

26. Goulet J, Lamarche B, Nadeau G, Lemieux S. Effect of a nutritional intervention promoting the Mediterranean food pattern on plasma lipids, lipoproteins and body weight in healthy French-Canadian women. Atherosclerosis 2003; 170: $115-24$.

27. Trichopoulou A, Costacou T, Bamia C, Trichopoulos D. Adherence to a Mediterranean diet and survival in a Greek population. N Engl J Med 2003; 348: 2599-608.

28. Serra-Majem L, Ribas L, Ngo J, Ortega RM, Garcia A, PerezRodrigo C, et al. Food, youth and the Mediterranean diet in
Spain. Development of KIDMED, Mediterranean Diet Quality Index in children and adolescents. Public Health Nutr 2004; 7: 931-5.

29. Mendez MA, Popkin BM, Jakszyn P, Berenguer A, Tormo MJ, Sanchez MJ, et al. Adherence to a Mediterranean diet is associated with reduced 3-year incidence of obesity. J Nutr 2006; 136: 2934-8.

30. Sanchez-Tainta A, Estruch R, Bullo M, Corella D, GomezGracia E, Fiol M, et al. Adherence to a Mediterranean-type diet and reduced prevalence of clustered cardiovascular risk factors in a cohort of 3,204 high-risk patients. Eur J Cardiovasc Prev Rehabil 2008; 15: 589-93.

31. Carter SJ, Roberts MB, Salter J, Eaton CB. Relationship between Mediterranean Diet Score and atherothrombotic risk: findings from the Third National Health and Nutrition Examination Survey (NHANES III), 1988-1994. Atherosclerosis 2010; 210: 630-6.

32. Gardener H, Wright CB, Gu Y, Demmer RT, Boden-Albala B, Elkind MS, et al. Mediterranean-style diet and risk of ischemic stroke, myocardial infarction, and vascular death: the Northern Manhattan Study. Am J Clin Nutr 2011; 94: 1458-64.

33. Martinez-Gonzalez MA, Garcia-Lopez M, Bes-Rastrollo M, Toledo E, Martinez-Lapiscina EH, Delgado-Rodriguez M, et al. Mediterranean diet and the incidence of cardiovascular disease: a Spanish cohort. Nutr Metab Cardiovasc Dis 2011; 21: 237-44.

34. Kesse-Guyot E, Ahluwalia N, Lassale C, Hercberg S, Fezeu L, Lairon D. Adherence to Mediterranean diet reduces the risk of metabolic syndrome: a 6-year prospective study. Nutr Metab Cardiovasc Dis 2013; 23: 677-83.

35. Marder K, Gu Y, Eberly S, Tanner CM, Scarmeas N, Oakes D, et al. Relationship of Mediterranean diet and caloric intake to phenoconversion in huntington disease. JAMA Neurol 2013; 70: 1382-8.

36. Pitsavos C, Panagiotakos DB, Chrysohoou C, Kokkinos PF, Skoumas J, Papaioannou I, et al. The effect of the combination of Mediterranean diet and leisure time physical activity on the risk of developing acute coronary syndromes, in hypertensive subjects. J Hum Hypertens 2002; 16: 517-24.

37. Panagiotakos DB, Pitsavos C, Chrysohoou C, Skoumas J, Tousoulis D, Toutouza M, et al. Impact of lifestyle habits on the prevalence of the metabolic syndrome among Greek adults from the ATTICA study. Am Heart J 2004; 147: 106-12.

38. Vincent-Baudry S, Defoort C, Gerber M, Bernard MC, Verger $\mathrm{P}$, Helal $\mathrm{O}$, et al. The Medi-RIVAGE study: reduction of cardiovascular disease risk factors after a 3-mo intervention with a Mediterranean-type diet or a low-fat diet. Am J Clin Nutr 2005; 82: 964-71.

39. Panagiotakos DB, Polystipioti A, Papairakleous N, Polychronopoulos E. Long-term adoption of a Mediterranean diet is associated with a better health status in elderly people; a cross-sectional survey in Cyprus. Asia Pac J Clin Nutr 2007; 16: 331-7.

40. Fung TT, Rexrode KM, Mantzoros CS, Manson JE, Willett WC, Hu FB. Mediterranean diet and incidence of and mortality from coronary heart disease and stroke in women. Circulation 2009; 119: 1093-100.

41. Hoevenaar-Blom MP, Nooyens AC, Kromhout D, Spijkerman AM, Beulens JW, van der Schouw YT, et al. Mediterranean style diet and 12-year incidence of cardiovascular diseases: the EPIC-NL cohort study. PLoS One 2012; 7: e45458.

42. Public Health Agency of Canada (2014). Economic burden of illness in Canada, 2005-2008. Ottawa, ON: Public Health Agency of Canada.

43. Canadian Institute for Health Information (2013). National Health Expenditure Trends, 1975 to 2013. Ottawa, ON: Canadian Institute for Health Information. 
44. Go AS, Mozaffarian D, Roger VL, Benjamin EJ, Berry JD, Blaha MJ, et al. Heart disease and stroke statistics-2014 update: a report from the American Heart Association. Circulation 2014; 129: e28-e292.

45. Drummond MF, Sculpher MJ, Torrance GW, O'Brien BJ, Stoddart GL (Eds.). Methods for the economic evaluation of health care programmes. 3rd ed. Oxford: Oxford University Press; 2005.

46. Public Health Agency of Canada (1998). Economic burden of illness in Canada, 1998. Ottawa, ON: Public Health Agency of Canada.

47. Roberts RR, Frutos PW, Ciavarella GG, Gussow LM, Mensah EK, Kampe LM, et al. Distribution of variable vs fixed costs of hospital care. JAMA 1999; 281: 644-9.

48. Williams RM. The costs of visits to emergency departments. N Engl J Med 1996; 334: 642-6.

49. Lave JR, Lave LB. Hospital cost functions. Annu Rev Public Health 1984; 5: 193-213.

50. Panagiotakos DB, Pitsavos C, Arvaniti F, Stefanadis C. Adherence to the Mediterranean food pattern predicts the prevalence of hypertension, hypercholesterolemia, diabetes and obesity, among healthy adults; the accuracy of the MedDietScore. Prev Med 2007; 44: 335-40.

51. Tangney CC, Kwasny MJ, Li H, Wilson RS, Evans DA, Morris MC. Adherence to a Mediterranean-type dietary pattern and cognitive decline in a community population. Am J Clin Nutr 2011; 93: 601-7.

52. Leblanc V, Begin C, Hudon AM, Royer MM, Corneau L, Dodin S, et al. Gender differences in the long-term effects of a nutritional intervention program promoting the Mediterranean diet: changes in dietary intakes, eating behaviors, anthropometric and metabolic variables. Nutr J 2014; 13: 107.

53. Zbeida M, Goldsmith R, Shimony T, Vardi H, Naggan L, Shahar DR. Mediterranean diet and functional indicators among older adults in non-Mediterranean and Mediterranean countries. J Nutr Health Aging 2014; 18: 411-18.

54. Pritchard C, Sculpher MJ. Productivity costs: principles and practice in economic evaluation. Office of Health Economics, London; 2000.

55. Brouwer WBF, Koopmanschap MA, Ruttenm FFH. Productivity costs in cost effectiveness analysis: numerator or denominator: a further discussion. Health Econ 1997; 6: 511-14.

56. Panagiotakos D, Sitara M, Pitsavos C, Stefanadis C. Estimating the 10-year risk of cardiovascular disease and its economic consequences, by the level of adherence to the Mediterranean diet: the ATTICA study. J Med Food 2007; 10: 239-43.

57. Schwingshackl L, Hoffmann G. Adherence to Mediterranean diet and risk of cancer: a systematic review and meta-analysis of observational studies. Int J Cancer 2014; 135: 1884-97.

58. Whitney EN, Rolfes SR. Understanding nutrition, 10th ed. Belmont, CA: Thomson Wadsworth; 2005.

59. Wansink B. From mindless eating to mindlessly eating better. Physiol Behav 2010; 100: 454-63.

*Peter J.H. Jones

Richardson Centre for Functional Foods and Nutraceuticals

196 Innovation Drive, SmartPark

University of Manitoba

Winnipeg, MB, Canada R3T 6C5

Email: peter_jones@umanitoba.ca 